\title{
A formula for the bivariate map asymptotics constants in terms of the univariate map asymptotics constants
}

\author{
Zhicheng Gao* \\ School of Mathematics and Statistics \\ Carleton University \\ Ottawa, Canada K1S 5B6
}

Submitted: Oct 18, 2010; Accepted: Nov 9, 2010; Published: Nov 19, 2010

Mathematics Subject Classification: 05C10, 05C30

\begin{abstract}
The parameters $t_{g}, p_{g}, t_{g}(r)$ and $p_{g}(r)$ appear in the asymptotics for a variety of maps on surfaces and embeddable graphs. In this paper we express $t_{g}(r)$ in terms of $t_{g}$ and $p_{g}(r)$ in terms of $p_{g}$.
\end{abstract}

\section{Introduction}

The concepts in this paragraph will be made precise in the following paragraphs. The parameters $t_{g}$ and $p_{g}$ arise in the univariate asymptotic enumeration of a variety of maps on surfaces and the parameters $t_{g}(r)$ and $p_{g}(r)$ arise in the corresponding bivariate asymptotics for maps as well as embeddable graphs. The original recursions for these parameters make it extremely difficult to compute them for higher genus surfaces. In contrast, the other parameters in the asymptotics are usually easily determined. Recently a simple recursion has been obtained for $t_{g}$ and another conjectured for $p_{g}$. In this paper, we obtain simple expressions for the bivariate parameters $t_{g}(r)$ and $p_{g}(r)$ in terms of the corresponding univariate parameters.

A map is a connected graph $G$ embedded in a surface $S$ (a closed 2-manifold) such that all components of $S-G$ are simply connected regions, which are called faces. Loops and multiple edges are allowed in $G$. A map is rooted if an edge is distinguished together with a direction on the edge and a side of the edge. The exact enumeration of various types of maps on the sphere (or, equivalently, the plane) was carried out by Tutte and his students (see [28] for a survey) in the 1960s via his device of rooting. Beginning in the 1980s, Tutte's approach was used for the asymptotic enumeration of maps on general surfaces $[3,4,9,11,16,17,18,19]$. A matrix integral approach was initiated by 't

*Research supported by NSERC 
Hooft (see [25] for various connections with quantum gravity, representation theory, and algebraic geometry). Let $T_{g}(n)\left(P_{g}(n)\right)$ be the number of rooted $n$-edge maps on the orientable surface of genus $g$ (non-orientable surface with $2 g$ cross-caps). In 1986 Bender and Canfield showed that, for each fixed $g$ and as $n \rightarrow \infty$,

$$
T_{g}(n) \sim t_{g} n^{5(g-1) / 2} 12^{n}, P_{g}(n) \sim p_{g} n^{5(g-1) / 2} 12^{n},
$$

where $t_{g}$ and $p_{g}$ are positive constants which can be computed by complicated recursions. In 1988 Bender and Wormald [11] derived similar asymptotic formulas for 2-connected maps in which the constants $t_{g}$ and $p_{g}$ also appear.

In 1993, the author [18] showed that many natural families of maps satisfy asymptotic formulas similar to (1) in which the same constants $t_{g}$ and $p_{g}$ appear in the coefficients. So in some sense $t_{g}$ and $p_{g}$ are universal constants. There is a nice connection between $t_{g}$ and Painlevé I ODE, and this connection seems to be well-known in the quantum physics community. However, there are doubts as to whether the proofs of the relevant results in the physics literature are mathematically rigorous. See, e.g., [25, Section 3.6] and [14, p. 29] for some related information. It is also worth mentioning that conjecture (74) stated in $[14$, p. 29] follows immediately from [19, Thm. 1.4].

Recently, using representation theory and KP-hierarchy, Goulden and Jackson [22] derived a remarkably simple recursion for the numbers of rooted triangulations of orientable surfaces. Let $C_{n, g}$ be the number of rooted $2 n$-face triangulations (or, by duality, $2 n$-vertex cubic maps) of an orientable surface of genus $g$. Define $H_{n, g}=(3 n+2) C_{n, g}$ for $n \geqslant 1, g \geqslant 0$, and

$$
H_{-1,0}=1 / 2, \quad H_{0,0}=2 \text { and } H_{-1, g}=H_{0, g}=0 \text { for } g \neq 0 .
$$

Goulden and Jackson [22] showed that, for $(n, g) \neq(-1,0)$,

$$
H_{n, g}=\frac{4(3 n+2)}{n+1}\left(n(3 n-2) H_{n-2, g-1}+\sum_{i=-1}^{n-1} \sum_{h=0}^{g} H_{i, h} H_{n-2-i, g-h}\right) .
$$

Bender et al. [7] used this recursion to derive a simple recursion for $t_{g}$ which leads to an asymptotic formula for $t_{g}$. This asymptotic formula for $t_{g}$ was used in [20] to settle a conjecture of 't Hooft about analyticity of free energy. Let

$$
f_{g}=24^{-3 / 2} 6^{g / 2} \Gamma\left(\frac{5 g-1}{2}\right) t_{g} .
$$

It was shown in [7] that

$$
f_{g}=\frac{\sqrt{6}}{96}(5 g-4)(5 g-6) f_{g-1}+6 \sqrt{6} \sum_{h=1}^{g-1} f_{h} f_{g-h}, \quad f_{0}=-\frac{\sqrt{6}}{72},
$$

and hence the generating function $f(z)=\sum_{g \geqslant 1} f_{g} z^{g}$ satisfies the following second order nonlinear ODE: (note there are two typos in the ODE given in [7])

$$
f(z)=6 \sqrt{6}(f(z))^{2}+\frac{\sqrt{6}}{96} z\left(25 z^{2} f^{\prime \prime}(z)+25 z f^{\prime}(z)-f(z)+\frac{\sqrt{6}}{72}\right) .
$$


Garoufalidis et al. [20] noticed that the above ODE is Painlevé I in disguise. More precisely, they noticed that

$$
a_{g}=-\frac{72}{\sqrt{6}}\left(\frac{2}{\sqrt{6}}\right)^{g} f_{g}=-2^{g-2} \Gamma\left(\frac{5 g-1}{2}\right) t_{g}
$$

satisfies the following recursion

$$
a_{g}=\frac{(5 g-4)(5 g-6)}{48} a_{g-1}-\frac{1}{2} \sum_{h=1}^{g-1} a_{h} a_{g-h}, \quad a_{0}=1,
$$

and the formal series $w(z)=\sum_{g \geqslant 0} a_{g} z^{-(5 g-1) / 2}$ satisfies the following Painlevé I:

$$
w^{\prime \prime}(z)=6 w^{2}(z)-6 z .
$$

This recursion was studied by Joshi and Kitaev [24] in the context of Painlevé I, and they derived the following full asymptotic expansion:

$$
a_{g} \sim \frac{S}{\pi} A^{-2 g+1 / 2} \Gamma(2 g-1 / 2)\left(1+\sum_{l \geqslant 1} \frac{\mu_{l} A^{l}}{\prod_{k=1}^{l}(2 g-k-1 / 2)}\right),
$$

where

$$
A=\frac{8 \sqrt{3}}{5}, \quad S=-\frac{1}{2 \sqrt{\pi}} 3^{1 / 4}
$$

and $\mu_{l}$ can be computed recursively using

$$
\mu_{l}=\frac{5}{16 \sqrt{3} l}\left(\frac{192}{25} \sum_{k=0}^{l-1} \mu_{k} a_{(l-k+1) / 2}-\left(l-\frac{9}{10}\right)\left(l-\frac{1}{10}\right) \mu_{l-1}\right), \quad \mu_{0}=1 .
$$

In the above (and below), it is understood that $a_{j}=0$ when $j$ is not an integer.

Based on evidence from quantum physics, Garoufalidis and Mariño [21] conjectured that

$$
p_{g}=\frac{1}{2^{g-2} \Gamma\left(\frac{5 g-3}{2}\right)} v_{2 g-1}
$$

where $v_{g}$ satisfies

$$
v_{g}=\frac{1}{2 \sqrt{3}}\left(-3 a_{g / 2}+\frac{5 g-6}{2} v_{g-1}+\sum_{k=1}^{g-1} v_{k} v_{g-k}\right),
$$

and $a_{j}$ is defined by (3). In [21], a nice asymptotic formula was also derived for $v_{g}$ using the above recursion for $v_{g}$ and the asymptotic expression for $a_{g}$. 
In $[5,12]$, interesting connections were shown between $t_{g}$ and the $g$ th moment of some random variables defined on trees.

In 1993, Bender, Canfield, and Richmond [4] derived a bivariate version of formula (1). Let $T_{g}(i, j)\left(P_{g}(i, j)\right)$ be the number of rooted maps, with $i$ faces and $j$ vertices, on the orientable surface of genus $g$ (non-orientable surface with $2 g$ cross-caps). They showed

$$
T_{g}(i, j) \sim t_{g}(r)(i j)^{5 g / 4-3 / 2} u_{0}^{-i} v_{0}^{-j}, \quad P_{g}(i, j) \sim p_{g}(r)(i j)^{5 g / 4-3 / 2} u_{0}^{-i} v_{0}^{-j}
$$

where

$$
u_{0}=\frac{r^{3}(2+r)}{4\left(1+r+r^{2}\right)^{2}}, v_{0}=\frac{1+2 r}{4\left(1+r+r^{2}\right)^{2}},
$$

and $r>0$ is determined by $j / i$ using the equation

$$
\frac{j}{i}=\frac{1+2 r}{r^{2}(2+r)} .
$$

For each $r>0, t_{g}(r)$ and $p_{g}(r)$ are positive constants which can be computed by complicated recursions (which are given in sections 2 and 3 below).

Our main result in this paper is the following.

\section{Theorem 1 Define}

$$
\begin{aligned}
& c(r)=\frac{r^{3}(1+2 r)(2+r)}{32 \sqrt{\pi}}\left(4+7 r+4 r^{2}\right)^{-1 / 2}\left(1+r+r^{2}\right)^{-7 / 2} \\
& d(r)=32 \sqrt{3} r^{-7 / 2}\left(1+r+r^{2}\right)^{4}(1+r)^{3 / 2}(2+r)^{-5 / 4}(1+2 r)^{-5 / 4}
\end{aligned}
$$

Then

$$
\begin{aligned}
t_{g}(r) & =c(r)[d(r)]^{g} t_{g} \\
p_{g}(r) & =c(r)[d(r)]^{g} p_{g} .
\end{aligned}
$$

We note that the above formulas easily lead to asymptotic formulas for $t_{g}(r)$ and $p_{g}(r)$ (as $g \rightarrow \infty$ ), using the corresponding asymptotic formulas for $t_{g}$ and $p_{g}$.

Finally we mention that $t_{g}(r)$ and $p_{g}(r)$ also appear in the asymptotic expressions for the numbers of 2-connected and 3-connected maps with $i$ faces and $j$ vertices [6]. Recently there have been considerable interest in enumerating graphs with a given genus (see, e.g., $[8,23,26,27])$. Let $G(S ; n)$ be the number of labelled graphs (no loops or multiple edges) with $n$ vertices which are embeddable in a surface $S$. In [26], McDiarmid established the exponential growth rate of $G(S ; n) / n$ ! by showing that, for each fixed surface $S$,

$$
\lim _{n \rightarrow \infty}(G(S ; n) / n !)^{1 / n}=\gamma
$$

for some positive constant $\gamma$ which is independent of $S$. The algebraic growth rate of $G(S ; n)$ was only established very recently. Bender and Gao [6] and Chapuy et al. [13] independently showed that

$$
G(S ; n) / n ! \sim c(S) n^{(5 g-7) / 2} \gamma^{n}, \quad(n \rightarrow \infty)
$$


where $g=1-\chi(S) / 2$ with $\chi(S)$ being the Euler characteristic of the surface $S$, and $c(S)$ is a positive constant depending on $S$. In [6], it was shown that

$$
c(S)= \begin{cases}A B^{g} t_{g}\left(r_{0}\right) & : \text { when } S \text { is the orientable surface of genus } g \\ A B^{g} p_{g}\left(r_{0}\right) & : \text { when } S \text { is the non-orientable surface with } 2 g \text { cross-caps }\end{cases}
$$

where $r_{0}, A$, and $B$ are positive constants which are independent of $S$. Furthermore, $t_{g}(r)$ and $p_{g}(r)$ also appear in the asymptotic expressions for the numbers of $k$-connected $(0 \leqslant k \leqslant 3)$ labelled graphs of genus $g$ with respect to vertices and edges.

Our approach is similar to that used in [18]. Using an appropriate normalizing factor, we can show that the complicated recursions satisfied by $t_{g}$ and $t_{g}(r)$ ( similarly for $p_{g}$ and $\left.p_{g}(r)\right)$ are equivalent. The main difference is that here we are comparing recursions for $t_{g}(r)\left(p_{g}(r)\right)$, which are bivariate in the sense that they involve a second parameter $r$, with the univariate recursions for $t_{g}\left(p_{g}\right)$, whereas in [18] all recursions are univariate. As a result, our normalizing factor used in this paper is slightly more sophisticated and involves the second parameter $r$.

\section{Connection between $t_{g}(r)$ and $t_{g}$}

In this section we prove Theorem 1 for orientable surfaces. Our approach will be similar to that used in [18]. We will show that the recursions satisfied by $t_{g}(r)$ can be normalized to match those satisfied by $t_{g}$. We need to recall some definitions and notation from $[3,4]$.

Let $\hat{M}_{g}(x, y, I)$ be the generating function for rooted maps on the orientable surface of genus $g$, where $x$ marks the number of edges, $y$ marks the root face degree, and each $z_{i}, i \in I$, marks the degree of the $i$ th distinguished face. For

$$
f=\frac{5-\sqrt{1-12 x}}{4+2 x}, \quad \boldsymbol{\alpha}=\left(\alpha_{i}\right)_{i \in I}, \quad \text { and } \quad|\boldsymbol{\alpha}|=\sum_{i \in I} \alpha_{i}
$$

define

$$
\hat{M}_{g}^{(n)}(x, I, \boldsymbol{\alpha})=\left.\frac{\partial^{n+|\boldsymbol{\alpha}|}}{\partial y^{n} \prod_{i \in I} \partial z_{i}^{\alpha_{i}}}\right|_{y=z_{i}=f} .
$$

We note that our $\hat{M}_{g}^{(n)}(x, I, \boldsymbol{\alpha})$ is the same as $\hat{H}_{g}^{(n)}(x, I, \boldsymbol{\alpha})$ used in [3].

In the following,

$$
F(x) \approx c\left(1-x / x_{0}\right)^{a} \quad\left(\text { as } x \rightarrow x_{0}\right)
$$

means that $F(x)$ is analytic in the region $\left.\left\{x:|x|<x_{0}+\delta\right\}-\left[x_{0}, x_{0}+\delta\right]\right\}$ for some small $\delta>0$, and it can be written as

$$
F(x)=p(x)+c\left(1-x / x_{0}\right)^{a}+o\left(\left(1-x / x_{0}\right)^{a}\right), \quad\left(\text { as } x \rightarrow x_{0}\right)
$$

where $p(x)$ is a polynomial in $x, x_{0}, c \neq 0$, and $a$ is not a non-negative integer.

We will also use $\emptyset$ to denote the empty set and $\mathbf{0}$ to denote the zero vector. For $J \subseteq I$, $\left.\boldsymbol{\alpha}\right|_{J}$ denotes the vector obtained by projecting $\boldsymbol{\alpha}$ onto $J$. 
It was shown in [3, Theorem 5] that

$$
\hat{M}_{g}^{(n)}(x, I, \boldsymbol{\alpha}) \approx \hat{\phi}_{g}^{(n)}(I, \boldsymbol{\alpha})(1-12 x)^{-(10 g+2 n+5|I|+2|\boldsymbol{\alpha}|-3) / 4}
$$

as $x \rightarrow 1 / 12$, where $\hat{\phi}_{g}^{(n)}(I, \boldsymbol{\alpha})$ satisfy recursion [3, (4.2)]. With $t=n+1$ and noting

$$
d_{t}=\frac{6}{125} \hat{\phi}_{0}^{(t)}(\emptyset, \mathbf{0}), \quad(t \geqslant 1)
$$

we can rewrite $[3,(4.2)]$ as the following recursion.

$$
\begin{aligned}
& -\left(\begin{array}{c}
n+1 \\
n
\end{array}\right) \hat{\phi}_{0}^{(1)}(\emptyset, \mathbf{0}) \hat{\phi}_{g}^{(n)}(I, \boldsymbol{\alpha}) \\
= & \left.\sum_{k=0}^{n-1}\left(\begin{array}{c}
n+1 \\
k
\end{array}\right) \hat{\phi}_{0}^{(n+1-k)}(\emptyset, \mathbf{0}) \hat{\phi}_{g}^{(k)}(I, \boldsymbol{\alpha})\right) \\
& +\frac{1}{2} \sum_{j=0}^{g} \sum_{J \subseteq I} \sum_{k=0}^{n+1}\left(\begin{array}{c}
n+1 \\
k
\end{array}\right) \hat{\phi}_{j}^{(k)}\left(J,\left.\boldsymbol{\alpha}\right|_{J}\right) \hat{\phi}_{g-j}^{(n+1-k)}\left(I-J,\left.\boldsymbol{\alpha}\right|_{I-J}\right) \\
& +\frac{3}{5} \sum_{k=0}^{n+1}\left(\begin{array}{c}
n+1 \\
k
\end{array}\right) \hat{\phi}_{g-1}^{(n+1-k)}\left(I+\{\omega\}, \boldsymbol{\alpha}+(k+1) e_{\omega}\right) \\
& +\frac{3}{5} \sum_{i \in I} \frac{(n+1) ! \alpha_{i} !}{\left(n+\alpha_{i}+2\right) !} \hat{\phi}_{g}^{\left(n+\alpha_{i}+2\right)}\left(I-\{i\},\left.\boldsymbol{\alpha}\right|_{I-\{i\}}\right)
\end{aligned}
$$

with the initial values

$$
\hat{\phi}_{0}^{(n)}(\emptyset, \mathbf{0})=5 \sqrt{6}\left(\frac{-25}{18}\right)^{n}\left(\begin{array}{c}
1 / 2 \\
n-1
\end{array}\right) n !
$$

Also

$$
t_{g}=\frac{1}{\Gamma((5 g-3) / 2)}\left(\frac{6}{25} \sum_{j=1}^{g-1} \hat{\phi}_{j}^{(0)}(\emptyset, \mathbf{0}) \hat{\phi}_{g-j}^{(0)}(\emptyset, \mathbf{0})+\frac{36}{125} \hat{\phi}_{g-1}^{(0)}\left(\{\omega\}, e_{\omega}\right)\right) .
$$

In the above (and the following) $e_{\omega}$ denotes the unit vector with a 1 in the $\omega$ th component (We note that in [3], $\omega \rightarrow 1$ was used for this purpose). We also note that the above recursion can be used, in the lexicographic order of $(g,|I|,|\boldsymbol{\alpha}|, n)$, to compute $\hat{\phi}_{g}^{(n)}(I, \boldsymbol{\alpha})$.

We now turn to the bivariate version of the above recursions.

Let $\hat{M}_{g}(u, v, y, I)$ be the bivariate analogy to $\hat{M}(x, y, I)$ with $u$ marking the number of faces and $v$ marking the number of vertices. Define

$$
\begin{aligned}
A(u, v, y)= & 1-y+u y^{2}+2 u^{-1} y^{2}(y-1) \hat{M}_{0}(u, v, y, \emptyset) \\
B(u, v, y)= & \left((1-p)^{2}\left(p^{2}+4 q^{2}\right)-4 q(1-p)^{3}\right) y^{4} \\
& +2\left(4 q(1-p)^{2}-(1-p)\left(p+4 q^{2}\right)\right) y^{3} \\
& +\left(1+4 q^{2}+(1-p)(2 p-4 q)\right) y^{2}-2 y+1,
\end{aligned}
$$


where

$$
u=p(1-p-2 q), v=q(1-2 p-q) .
$$

It was shown in [4] that $\hat{M}_{0}(u, v, y, \emptyset)$ satisfies $A^{2}=B$, and for $g>0, \hat{M}_{g}(u, v, y, I)$ is determined by the following recursion

$$
\begin{aligned}
& A(u, v, y) \hat{M}_{g}(u, v, y, I) \\
= & -\frac{y^{2}(y-1)}{u} \sum_{\substack{j=0 \\
(j, J) \neq(0, \emptyset),(g, I)}} \hat{M}_{j}(u, v, y, J) \hat{M}_{g-j}(u, v, y, I-J) \\
& -\left.\frac{y^{3}(y-1)}{u} \frac{\partial}{\partial z_{w}} \hat{M}_{g-1}(u, v, y, I+\{\omega\})\right|_{z_{\omega}=y} \\
& -u y(y-1) \sum_{i \in I} \frac{z_{i}}{z_{i}-y}\left[z_{i} \hat{M}_{g}\left(u, v, z_{i}, I-\{i\}\right)-y \hat{M}_{g}(u, v, y, I-\{i\})\right] \\
& +u y \hat{M}_{g}(u, v, 1, I) .
\end{aligned}
$$

We note that this is the orientable analogy to $[4,(4.1)]$.

Let the parameters $r$ and $s$ be related to $p$ and $q$ by

$$
p=\frac{r}{2(1+r+s)}, \quad q=\frac{s}{2(1+r+s)} .
$$

Then

$$
u=\frac{r(2+r)}{4(1+r+s)^{2}}, v=\frac{s(2+s)}{4(1+r+s)^{2}} .
$$

Let

$$
y_{0}=\frac{2\left(1+r+r^{2}\right)}{2+2 r+r^{2}}
$$

$u_{0}$ be as defined in (6), and

$$
B^{(n)}=\left.\frac{\partial^{n} B(u, v, y)}{\partial y^{n}}\right|_{y=1 /(1-p)}
$$

It follows from $[4,(2.4)]$ and the expressions for $B^{(n)}, n=2,3$, on page 328 of [4] that

$$
\begin{aligned}
& B^{(0)}=B^{(1)}=0, \\
& B^{(2)}=\frac{2(1-r s)}{(1+r+s)^{2}}=c_{2}\left(1-u / u_{0}\right)^{1 / 2}+O\left(1-u / u_{0}\right), \\
& B^{(3)}=-12(1-p)(p(1-2 p)+4 q(1-p-q))=-c_{3}+O\left(\left(1-u / u_{0}\right)^{1 / 2}\right),
\end{aligned}
$$

as $u \rightarrow u_{0}$, where

$$
c_{2}=\frac{2 r^{2}}{\left(1+r+r^{2}\right)^{2}} \sqrt{3(2+r)(1+r)}, \quad c_{3}=\frac{3(1+r)\left(2+2 r+r^{2}\right)^{2}}{\left(1+r+r^{2}\right)^{3}} .
$$


The following results were implicitly used in [4]. For the readers who are not familiar with $[3,4]$, we briefly outline how they are derived from (14). As in the univariate case, we define

$$
\hat{M}_{g}^{(n)}(u, v, I, \boldsymbol{\alpha})=\left.\frac{\partial^{n+|\boldsymbol{\alpha}|}}{\partial y^{n} \prod_{i \in I} \partial z_{i}^{\alpha_{i}}} \hat{M}_{g}(u, v, y, I)\right|_{y=z_{i}=1 /(1-p)} .
$$

Using the above singular expansions of $B^{(2)}$ and $B^{(3)}$, and the same argument used in the proof of [3, Lemma 2], we obtain

$$
\hat{M}_{0}^{(n)}(u, v, \emptyset, \mathbf{0}) \approx \frac{3 c_{2} u_{0}}{2 c_{3} y_{0}^{2}\left(y_{0}-1\right)} \sqrt{\frac{c_{2}}{2}}\left(-\frac{c_{3}}{3 c_{2}}\right)^{n}\left(\begin{array}{c}
1 / 2 \\
n-1
\end{array}\right) n !\left(1-u / u_{0}\right)^{-(2 n-3) / 4},
$$

where the factor $\frac{u_{0}}{2 y_{0}^{2}\left(y_{0}-1\right)}$ comes from the coefficient of $\hat{M}_{0}(u, v, y, \emptyset)$ in (12).

Applying

$$
\left.\frac{\partial^{n+1+|\boldsymbol{\alpha}|}}{\partial y^{n+1} \prod_{i \in I} \partial z_{i}^{\alpha_{i}}}\right|_{y=z_{i}=1 /(1-p)}
$$

to both sides of (14), we obtain (by induction on the lexicographic order of $(g,|I|,|\boldsymbol{\alpha}|, n))$,

$$
\hat{M}_{g}^{(n)}(u, v, I, \boldsymbol{\alpha}) \approx \hat{M}_{g}^{(n)}(I, \boldsymbol{\alpha})\left(1-u / u_{0}\right)^{-(10 g+2 n+5|I|+2|\boldsymbol{\alpha}|-3) / 4}
$$

as $u \rightarrow u_{0}$, where $\hat{M}_{g}^{(n)}(I, \boldsymbol{\alpha})$ satisfy the following recursion:

$$
\begin{aligned}
& -\left(\begin{array}{c}
n+1 \\
n
\end{array}\right) \hat{M}_{0}^{(1)}(\emptyset, \mathbf{0}) \hat{M}_{g}^{(n)}(I, \boldsymbol{\alpha}) \\
= & \left.\sum_{k=0}^{n-1}\left(\begin{array}{c}
n+1 \\
k
\end{array}\right) \hat{M}_{0}^{(n+1-k)}(\emptyset, \mathbf{0}) \hat{M}_{g}^{(k)}(I, \boldsymbol{\alpha})\right) \\
& +\frac{1}{2} \sum_{j=0}^{g} \sum_{J \subseteq I} \sum_{k=0}^{n+1}\left(\begin{array}{c}
n+1 \\
k
\end{array}\right) \hat{M}_{j}^{(k)}\left(J,\left.\boldsymbol{\alpha}\right|_{J}\right) \hat{M}_{g-j}^{(n+1-k)}\left(I-J,\left.\boldsymbol{\alpha}\right|_{I-J}\right) \\
& +\frac{y_{0}}{2} \sum_{k=0}^{n+J}\left(\begin{array}{c}
n+1 \\
k
\end{array}\right) \hat{M}_{g-1}^{(n+1-k)}\left(I+\{\omega\}, \boldsymbol{\alpha}+(k+1) e_{\omega}\right) \\
& +\frac{u_{0}^{2} y_{0}}{2} \sum_{i \in I} \frac{(n+1) ! \alpha_{i} !}{\left(n+\alpha_{i}+2\right) !} \hat{M}_{g}^{\left(n+\alpha_{i}+2\right)}\left(I-\{i\},\left.\boldsymbol{\alpha}\right|_{I-\{i\}}\right) .
\end{aligned}
$$

Define

$$
\beta_{0}=\frac{u_{0} c_{2} \sqrt{3 c_{2}}}{20 c_{3} y_{0}^{2}\left(y_{0}-1\right)}, \beta_{1}=\frac{6 c_{3}}{25 c_{2}}, \beta_{2}=\frac{5 u_{0} y_{0} \beta_{1}}{6 \beta_{0}}, \beta_{3}=u_{0} \beta_{2} .
$$

Then it is not difficult to check that recursions (9) and (18) are equivalent under the transformation

$$
\hat{M}_{g}^{(n)}(I, \boldsymbol{\alpha})=\beta_{0} \beta_{1}^{n+|\boldsymbol{\alpha}|} \beta_{2}^{2 g} \beta_{3}^{|I|} \hat{\phi}_{g}^{(n)}(I, \boldsymbol{\alpha}) .
$$


Their initial values (10) and (17) are also equivalent under this transformation. Thus we have, for all $g, n, I, \boldsymbol{\alpha}$, that

$$
\hat{M}_{g}^{(n)}(I, \boldsymbol{\alpha})=\beta_{0} \beta_{1}^{n+|\boldsymbol{\alpha}|} \beta_{2}^{2 g} \beta_{3}^{|I|} \hat{\phi}_{g}^{(n)}(I, \boldsymbol{\alpha}) .
$$

Setting $y=\frac{1}{1-p}$ and $I=\emptyset$ in $(14)$, we obtain

$$
\begin{aligned}
\hat{M}_{g}\left(u, v_{0}, 1, \emptyset\right) & \approx\left(\frac{y_{0}\left(y_{0}-1\right)}{u_{0}^{2}} \sum_{j=1}^{g-1} \hat{M}_{j}^{(0)}(\emptyset, \mathbf{0}) \hat{M}_{g-j}^{(0)}(\emptyset, \mathbf{0})\right. \\
& \left.+\frac{y_{0}^{2}\left(y_{0}-1\right)}{u_{0}^{2}} \hat{M}_{g-1}^{(0)}\left(\{\omega\}, e_{\omega}\right)\right)\left(1-u / u_{0}\right)^{-(5 g-3) / 2},
\end{aligned}
$$

as $u \rightarrow u_{0}$.

Using the Flajolet-Odlyzko "transfer theorem" [15, Corollary VI.1], (11) and (20), we obtain

$$
\begin{aligned}
{\left[u^{i}\right] \hat{M}_{g}(u, v, 1, \emptyset) } & \sim \frac{1}{\Gamma((5 g-3) / 2)}\left(\frac{y_{0}\left(y_{0}-1\right)}{u_{0}^{2}} \sum_{j=1}^{g-1} \hat{M}_{j}^{(0)}(\emptyset, \mathbf{0}) \hat{M}_{g-j}^{(0)}(\emptyset, \mathbf{0})\right. \\
& \left.+\frac{y_{0}^{2}\left(y_{0}-1\right)}{u_{0}^{2}} \hat{M}_{g-1}^{(0)}\left(\{w\}, e_{w}\right)\right) i^{5(g-1) / 2} u_{0}^{-i} \\
& =\frac{25 y_{0}\left(y_{0}-1\right)}{6 u_{0}^{2}} \beta_{0}^{2} \beta_{2}^{2 g} t_{g} i^{5(g-1) / 2} u_{0}^{-i}
\end{aligned}
$$

as $i \rightarrow \infty$ uniformly for $r$ in any closed subinterval of $(0, \infty)$.

As indicated in [4], the local limit theorem [10] gives

$$
T_{g}(i, j)=\left[u^{i} v^{j}\right] \hat{M}_{g}(u, v, 1, \emptyset) \sim \frac{25 y_{0}\left(y_{0}-1\right)}{6 u_{0}^{2} \sigma \sqrt{i 2 \pi}} \beta_{0}^{2} \beta_{2}^{2 g} t_{g} i^{5(g-1) / 2} u_{0}^{-i} v_{0}^{-j}
$$

with [4, Lemma 3]

$$
\frac{j}{i}=\frac{1+2 r}{r^{2}(2+r)}, \quad \sigma^{2}=\frac{(1+2 r)\left(1+r+r^{2}\right)\left(4+7 r+4 r^{2}\right)}{6 r^{4}(1+r)(2+r)^{2}} .
$$

This gives the first asymptotic expression in (5) with

$$
t_{g}(r)=\frac{25 y_{0}\left(y_{0}-1\right)}{6 u_{0}^{2} \sigma \sqrt{2 \pi}} \beta_{0}^{2} \beta_{2}^{2 g}\left(\frac{r^{2}(2+r)}{1+2 r}\right)^{(5 g-6) / 4} t_{g} .
$$

Now (4) follows from (6), (15), (19), (22), and (23). Using $t_{0}=2 / \sqrt{\pi}$ and $t_{1}=1 / 24$ [3], we can verify that our expressions for $t_{0}(r)$ and $t_{1}(r)$ agree with those given in [4, Theorem 1]. 


\section{Connection between $p_{g}(r)$ and $p_{g}$}

In this section, we provide the proof to Theorem 1 for non-orientable surfaces. Since the argument is essentially the same as the one used in the previous section for orientable surfaces, we will just outline where the minor differences are.

In analogy to the orientable case in section 2 , let $M_{g}(x, y, I)\left(M_{g}(u, v, y, I)\right)$ be the generating function for rooted maps with respect edges (faces and vertices) on a surface (orientable or non-orientable) of Euler characteristic $2-2 g$. Hence the surface is either orientable of genus $g$, or non-orientable with $2 g$ cross-caps. Then

$$
T_{g}(n)+P_{g}(n)=\left[x^{n}\right] M_{g}(x, 1, \emptyset), \quad T_{g}(i, j)+P_{g}(i, j)=\left[u^{i} v^{j}\right] M_{g}(u, v, 1, \emptyset) .
$$

It is known $[3,(3.6)]$ that

$$
\begin{aligned}
t_{g}+p_{g}= & \frac{1}{\Gamma((5 g-3) / 2)}\left(\frac{6}{25} \sum_{j=1 / 2}^{g-1 / 2} \phi_{j}^{(0)}(\emptyset, \mathbf{0}) \phi_{g-j}^{(0)}(\emptyset, \mathbf{0})\right. \\
& \left.+\frac{72}{125} \phi_{g-1}^{(0)}\left(\{\omega\}, e_{\omega}\right)+\frac{36}{125} \phi_{g-1 / 2}^{(1)}(\emptyset, \mathbf{0})\right)
\end{aligned}
$$

where the constants $\left.\phi_{g}^{(k)}(I, \boldsymbol{\alpha})\right)$ satisfy the following recursion (noting the remark before $(10))$.

$$
\begin{aligned}
& -\left(\begin{array}{c}
n+1 \\
n
\end{array}\right) \phi_{0}^{(1)}(\emptyset, \mathbf{0}) \phi_{g}^{(n)}(I, \boldsymbol{\alpha}) \\
= & \left.\sum_{k=0}^{n-1}\left(\begin{array}{c}
n+1 \\
k
\end{array}\right) \phi_{0}^{(n+1-k)}(\emptyset, \mathbf{0}) \phi_{g}^{(k)}(I, \boldsymbol{\alpha})\right) \\
& +\frac{1}{2} \sum_{j=0 / 2}^{g} \sum_{J \subseteq I} \sum_{k=0}^{n+1}\left(\begin{array}{c}
n+1 \\
k
\end{array}\right) \phi_{j}^{(k)}\left(J,\left.\boldsymbol{\alpha}\right|_{J}\right) \phi_{g-j}^{(n+1-k)}\left(I-J,\left.\boldsymbol{\alpha}\right|_{I-J}\right) \\
& +\frac{6}{5} \sum_{k=0}^{n+1}\left(\begin{array}{c}
n+1 \\
k
\end{array}\right) \phi_{g-1}^{(n+1-k)}\left(I+\{\omega\}, \boldsymbol{\alpha}+(k+1) e_{\omega}\right) \\
& +\frac{3}{5} \phi_{g-1 / 2}^{(n+2)}(I, \boldsymbol{\alpha}) \\
& +\frac{3}{5} \sum_{i \in I} \frac{(n+1) ! \alpha_{i} !}{\left(n+\alpha_{i}+2\right) !} \phi_{g}^{\left(n+\alpha_{i}+2\right)}\left(I-\{i\},\left.\boldsymbol{\alpha}\right|_{I-\{i\}}\right)
\end{aligned}
$$

with the initial values given by

$$
\phi_{0}^{(n)}(\emptyset, \mathbf{0})=\hat{\phi}_{0}^{(0)}(\emptyset, \mathbf{0}) . \quad(\operatorname{as} \text { in }(10))
$$


We note, in here and below, the summation for $j$ from $0 / 2$ indicates that $j$ is over all the half integers in the specified range.

As in the previous section, we obtain from $[4,(4.1)]$ that

$$
\begin{aligned}
& -\left(\begin{array}{c}
n+1 \\
n
\end{array}\right) M_{0}^{(1)}(\emptyset, \mathbf{0}) M_{g}^{(n)}(I, \boldsymbol{\alpha}) \\
= & \left.\sum_{k=0}^{n-1}\left(\begin{array}{c}
n+1 \\
k
\end{array}\right) M_{0}^{(n+1-k)}(\emptyset, \mathbf{0}) M_{g}^{(k)}(I, \boldsymbol{\alpha})\right) \\
& +\frac{1}{2} \sum_{j=0 / 2}^{g} \sum_{J \subseteq I} \sum_{k=0}^{n+1}\left(\begin{array}{c}
n+1 \\
k
\end{array}\right) M_{j}^{(k)}\left(J,\left.\boldsymbol{\alpha}\right|_{J}\right) M_{g-j}^{(n+1-k)}\left(I-J,\left.\boldsymbol{\alpha}\right|_{I-J}\right) \\
& +y_{0} \sum_{k=0}^{n+1}\left(\begin{array}{c}
n+1 \\
k
\end{array}\right) M_{g-1}^{(n+1-k)}\left(I+\{\omega\}, \boldsymbol{\alpha}+(k+1) e_{\omega}\right) \\
& +\frac{u_{0} y_{0}}{2} M_{g-1 / 2}^{(n+2)}(I, \boldsymbol{\alpha}) \\
& +\frac{u_{0}^{2} y_{0}}{2} \sum_{i \in I} \frac{(n+1) ! \alpha_{i} !}{\left(n+\alpha_{i}+2\right) !} M_{g}^{\left(n+\alpha_{i}+2\right)}\left(I-\{i\},\left.\boldsymbol{\alpha}\right|_{I-\{i\}}\right) .
\end{aligned}
$$

Let $\beta_{0}, \beta_{1}, \beta_{2}, \beta_{3}$ be as defined in (19), it is easy to verify that

$$
\beta_{0} \beta_{1}^{n+|\boldsymbol{\alpha}|} \beta_{2}^{2 g} \beta_{3}^{|I|} \phi_{g}^{(n)}(I, \boldsymbol{\alpha})
$$

satisfy (26), and hence

$$
\left.M_{g}^{(n)}(I, \boldsymbol{\alpha})\right)=\beta_{0} \beta_{1}^{n+|\boldsymbol{\alpha}|} \beta_{2}^{2 g} \beta_{3}^{|I|} \phi_{g}^{(n)}(I, \boldsymbol{\alpha}) .
$$

As in the previous section, this implies that

$$
\begin{aligned}
{\left[u^{i}\right] M_{g}(u, v, 1, \emptyset) \sim } & \frac{1}{\Gamma((5 g-3) / 2)}\left(\frac{y_{0}\left(y_{0}-1\right)}{u_{0}^{2}} \sum_{j=1 / 2}^{g-1 / 2} M_{j}^{(0)}(\emptyset, \mathbf{0}) M_{g-j}^{(0)}(\emptyset, \mathbf{0})\right. \\
& +\frac{2 y_{0}^{2}\left(y_{0}-1\right)}{u_{0}^{2}} M_{g-1}^{(0)}\left(\{\omega\}, e_{\omega}\right) \\
& \left.+\frac{y_{0}^{2}\left(y_{0}-1\right)}{u_{0}} M_{g-1 / 2}^{(1)}(\emptyset, \mathbf{0})\right) i^{5(g-1) / 2} u_{0}^{-i} \\
= & \frac{25 y_{0}\left(y_{0}-1\right)}{6 u_{0}^{2}} \beta_{0}^{2} \beta_{2}^{2 g}\left(t_{g}+p_{g}\right) i^{5(g-1) / 2} u_{0}^{-i} .
\end{aligned}
$$

Again, as indicated in [4], the local limit theorem gives

$$
T_{g}(i, j)+P_{g}(i, j) \sim \frac{25 y_{0}\left(y_{0}-1\right)}{6 u_{0}^{2} \sigma \sqrt{i 2 \pi}} \beta_{0}^{2} \beta_{2}^{2 g}\left(t_{g}+p_{g}\right) i^{5(g-1) / 2} u_{0}^{-i} v_{0}^{-j},
$$


and hence

$$
t_{g}(r)+p_{g}(r)=\frac{25 y_{0}\left(y_{0}-1\right)}{6 u_{0}^{2} \sigma \sqrt{2 \pi}} \beta_{0}^{2} \beta_{2}^{2 g}\left(\frac{r^{2}(2+r)}{1+2 r}\right)^{(5 g-6) / 4}\left(t_{g}+p_{g}\right) .
$$

This together with (23) gives

$$
p_{g}(r)=\frac{25 y_{0}\left(y_{0}-1\right)}{6 u_{0}^{2} \sigma \sqrt{2 \pi}} \beta_{0}^{2} \beta_{2}^{2 g}\left(\frac{r^{2}(2+r)}{1+2 r}\right)^{(5 g-6) / 4} p_{g} .
$$

Now (5) follows from (6), (15), (19), (22), and (28). This completes the proof of Theorem 1. Using $p_{1 / 2}=-2 \sqrt{6} / \Gamma(-1 / 4)$, we can verify that our expression for $p_{1 / 2}(r)$ agrees with that given in $[4$, Theorem 1$]$.

\section{Concluding remarks}

In this paper, we derived a simple expression for the coefficients $t_{g}(r)\left(p_{g}(r)\right)$ in the asymptotic formula for the number of rooted maps on an orientable (non-orientable) surface with Euler characteristic $2-2 g$, with respect to faces and vertices. As shown in Theorem $1, t_{g}(r)=c(r)[d(r)]^{g} t_{g}$ for some simple algebraic functions $c(r)$ and $d(r)$. Since $t_{g}$ can be efficiently computed using (3), so can $t_{g}(r)$. Furthermore, the asymptotic expression for $t_{g}$ leads to an asymptotic expression for $t_{g}(r)$. Also if the conjecture (4) of Garoufalidis and Mariño is true, then both $p_{g}$ and $p_{g}(r)$ can be efficiently computed. This implies that the coefficients in the asymptotic formulas for many families of maps and graphs can be computed efficiently.

We also mention that some results are known for computing the exact values of $T_{g}(n), P_{g}(n), T_{g}(i, j)$ and $P_{g}(i, j)$. For example, Arquès and Giorgetti $[1,2]$ showed

$$
\begin{aligned}
\sum_{i, j \geqslant 1} T_{g}(i, j) u^{i} v^{j} & =\frac{p q(1-p-q) \hat{Q}_{g}(p, q)}{\left[(1-2 p-2 q)^{2}-4 p q\right]^{5 g-3}}, \\
\sum_{i, j \geqslant 1}\left(T_{g}(i, j)+P_{g}(i, j)\right) u^{i} v^{j} & =\frac{Q_{g}(p, q, t)}{\left[(1-2 p-2 q)^{2}-4 p q\right]^{5 g-3}},
\end{aligned}
$$

where $\hat{Q}_{g}(p, q)$ is a polynomial in $p, q$ with total degree at most $6 g-3$, and $Q_{g}(p, q, t)$ is a polynomial in $p, q$, and $t=\sqrt{(1-2 p-2 q)^{2}-4 p q}$ with total degree at most $6 g-6$.

Since the above results were obtained using complicated recursions like (14), so far there is no efficient way known for computing $\hat{Q}_{g}(p, q)$ and $Q_{g}(p, q, t)$. In view of $(2)$, there might be simple recursions for $T_{g}(n)$ and $P_{g}(n)$, or even for $T_{g}(i, j)$ and $P_{g}(i, j)$. Indeed, it will be very interesting to find such simple recursions. 


\section{References}

[1] D. Arquès and Giorgetti, Enumeration des cartes pointées sur une surface orientable de genre quelconque en fonction des nombre de sommets et de faces, J. Combin. Theory Ser. B 77 (1999), 1-24.

[2] D. Arquès and Giorgetti, Countin rooted maps on a surface, Theoret. Comput. Sci. 234 (2000), 255-272.

[3] E.A. Bender and E.R. Canfield, The asymptotic number of rooted maps on a surface, J. Combin. Theory Ser. A 43 (1986), 244-257.

[4] E.A. Bender, E.R. Canfield and L.B. Richmond, The asymptotic number of rooted maps on a surface. II. Enumeration by vertices and faces, J. Combin. Theory Ser. A 63 (1993), no. 2, 318-329.

[5] E.A. Bender, A.B. Olde Daalhuis, Z. Gao, L.B. Richmond and N. C. Wormald, Asymptotics of Some Convolutional Recurrences, Electron. J. Combin. 17(1) (2010) R1, 11pp.

[6] E.A. Bender and Z. Gao, Asymptotic enumeration of labelled graphs with a given genus, http://arxiv.org/abs/0912.4670 (2009).

[7] E.A. Bender, Z. Gao and L.B. Richmond, The map asymptotics constant $t_{g}$, Electron. J. Combin. 15(1) (2008) R51, 8pp.

[8] E.A. Bender, Z. Gao, and N.C. Wormald, The number of labeled 2-connected planar graphs, Electron. J. Combin. 9 (2002) R43.

[9] E.A. Bender, Z. Gao, L.B. Richmond and N. C. Wormald, Asymptotic properties of rooted 3-connected maps on surfaces. J. Austral. Math. Soc. Ser. A 60 (1996), 31-41.

[10] E.A. Bender and L.B. Richmond, Central and local limit theorems applied to asymptotic enumeration II: Multivariate generating functions. J. Combin. Theory Ser. A $34255-265$.

[11] E.A. Bender and N.C. Wormald, The asymptotic number of rooted nonseparable maps on a surface, J. Combin. Theory Ser. A 49 (1988), no. 2, 370-380.

[12] G. Chapuy, The structure of unicellular maps, and a connection between maps of positive genus and planar labelled trees, Probability Theory and Related Fields 147 (2010), no. 3, 415-447.

[13] G. Chapuy, É. Fusy, O. Gimenez, B. Mohar and M. Noy, Asymptotic enumeration and limit laws for graphs of fixed genus, http://arxiv.org/abs/1001.3628 (2010).

[14] A.S. Fokas, A.R. Its, A.A. Kapaeve, and V.Y. Novokshenov, Painleve Transcendents: The Riemann-Hilbert Approach, Amer. Math Soc. 2006.

[15] P. Flajolet and R. Sedgewick, Analytic Combinatorics, Cambridge University Press, 2009.

[16] Z. Gao, The Number of Rooted Triangular Maps on a Surface, J. Combin. Theory, Ser. B 52 (1991), 236-249. 
[17] Z. Gao, The Asymptotic Number of Rooted 2-Connected Triangular Maps on a Surface, J. Combin. Theory, Ser. B 54 (1992), 102-112.

[18] Z. Gao, A Pattern for the Asymptotic Number of Rooted Maps on Surfaces, J. Combin. Theory Ser. A 64 (1993), 246-264.

[19] Z. Gao, The Number of Degree Restricted Maps on a Surface, Discrete Math. 123 (1993), 47-63.

[20] S. Garoufalidis, T. T. Lê, and M. Mariño, Analyticity of the free energy of a closed 3-manifold, SIGMA 4 (2008), 080, 20pp.

[21] S. Garoufalidis and M. Mariño, Universality and asymptotics of graph counting problems in unoriented surfaces, J. Combin. Theory Ser. A, 117 (2010), 715-740.

[22] I. Goulden and D.M. Jackson, The KP hierarchy, branched covers and triangulations, Adv. in Math. 219, (2008), 932-951.

[23] O. Giménez and M. Noy, Asymptotic enumeration and limit laws of planar graphs, J. Amer. Math. Soc. 22 (2009), 309-329.

[24] N. Joshi and A.V. Kitaev, On Boutroux's Tritronquée Solutions of the First Painlevé Equation, Stud. Appl. Math. 107 (2001), 253-291.

[25] S.K. Lando and A.K. Zvonkin, Graphs on Surfaces and Their Applications, volume 141 of Encyclopedia of Mathematical Mathematical Sciences, Spinger-Verlag, Berlin, 2004.

[26] C. McDiarmid, Random graphs on surfaces, J. Combin. Theory Ser. B 98 (2008), 778-797.

[27] C. McDiarmid, A. Steger, and D. Welsh, Random Planar Graphs, J. Combin. Theory Ser. B 93 (2005), 187-205.

[28] W.T. Tutte, The enumerative theory of planar maps. A Survey of Combinatorial Theory (Proc. Internat. Sympos., Colorado State Univ., Fort Collins, Colo., 1971), pp. 437-448. North-Holland, Amsterdam, 1973. 